\title{
Study of the clinico-etiological profile of fungal keratitis in a tertiary care hospital in North India
}

\author{
Prerna Upadhyay $^{1}$, Neeti Gupta ${ }^{2, *}$, Harsh Bahadur $^{3,}$ Aarti Kotwal $^{4}$ \\ ${ }^{1}$ Senior Resident, ${ }^{\mathbf{2}}$ Associate Professor, ${ }^{\mathbf{3}, \mathbf{4}}$ Professor, ${ }^{\mathbf{1}, \mathbf{2}, \mathbf{3}}$ Dept. of Ophthalmology, ${ }^{\mathbf{4}}$ Dept. of Microbiology, Himalayan Institute of \\ Medical Sciences, Dehradun, Uttarakhand, India \\ *Corresponding Author: \\ Email: drneetigupta@rediffmail.com
}

\begin{abstract}
Introduction: Given the rise in cases of fungal keratitis in recent years, this study was performed to better elucidate the clinical and microbiological profile, risk factors and intervention rates of fungal keratitis at a tertiary referral center in Uttarakhand.

Methods: Corneal scraping was collected from 53 consecutive cases of suspected fungal keratitis and was subjected to direct staining and culture.

Results: This was a prospective observational study conducted over a period of 1 year after approval from the institutional ethical committee. All the patients which appeared to be fungal or mixed keratitis on clinical examination were included in this study. Out of 53 patients, 37 (69.8\%) were males and 16 (30.2\%) females with a mean age of $46.04 \pm 16.23$ years. Maximum patients $18(33.96 \%)$ had the onset of symptoms and signs in the months of July-September. Of all the corneal ulcer which appeared fungal on clinical examination, $\mathrm{KOH}$ and culture was positive in $50.9 \%$ patients. While $18.9 \%$ were mixed type having both $\mathrm{KOH}$ and Gram stain positive. Out of 53 patients, 23 had presence of 3 or more typical clinical features of fungal keratits. There was significant association between number of clinical features and $\mathrm{KOH}$ and culture positivity. Fusarium and Curvularia species were the most common etiological agents in our region.

Conclusions: Predominant outdoor agricultural activity and trauma with vegetative matter was the principal causative factor for corneal injury. Corneal ulcers complicated due to prior treatment by village healers and hence another important concern. The information regarding regional etiology will help empirical management as many eye clinics do not have microbiological facilities.
\end{abstract}

Keywords: Cornea, Fungal keratitis, Microbiology.

\section{Introduction}

Corneal blindness is one of the important cause of blindness, especially in developing countries. ${ }^{1}$ According to the World Health Organization, corneal diseases are among the major causes of vision loss and blindness in the world today, after cataract and glaucoma. ${ }^{2}$

In India, it is estimated that there are approximately 6.8 million people who are blind due to corneal diseases and have vision less than $6 / 60$ in at least one eye. Around 1 million are blind due to bilateral corneal involvement. It is expected that the number of individuals with unilateral corneal blindness in India will increase to 10.6 million by $2020 .{ }^{3}$

Microbial keratitis is an important cause of corneal blindness in developing countries and can be due to bacteria, virus, fungi or parasites. Though there could be distinct clinical features that are specific to each of the agent groups, a clinical diagnosis can often be difficult due to overlapping features. ${ }^{4}$ Also, causes of microbial keratitis varies between different geographic area and varies between continents and countries. ${ }^{5}$ Fungal keratitis is difficult to treat due to low penetration of anti-fungal eye drops and resistance of these organisms towards medication. Thus, it is essential to determine the local etiological factor within a given region when planning a strategy for corneal ulcer. Early diagnosis and treatment can save the eye from becoming blind. ${ }^{6}$

\section{Aims and Objectives}

To study the clinico-etiological profile of fungal keratitis.

\section{Methods}

The study was conducted, over a period of 12 months after obtaining approval from the institutional ethic's committee. Patients attending department of ophthalmology with signs and symptoms suggestive of fungal keratitis or mixed infection were included in the study. Patients were excluded who were diagnosed with pure bacterial or viral or acanthamoeba keratitis. Patient with history of steroid treated keratitis or patient who were non-compliant or did not give consent were excluded from the study.

The socio demographic data, predisposing risk factors, clinical details, prior treatment modalities, laboratory results and clinical diagnosis were recorded in all cases. A detailed history was taken including mode, duration and course of the disease. Visual acuity was recorded on Snellen's chart. Detailed slit lamp examination was done in all cases. Corneal examination was performed in detail, noting the size of ulcer, depth and extent of infiltrations, presence/ absence of hypopyon and scleral extension. The ulcer was graded as mild, moderate or severe as per the Jones guidelines. ${ }^{6}$ Corneal scrapings for $\mathrm{KOH}$, grams stain 
and fungal culture was done on sabouraud dextrose agar (SDA).

For statistical analysis, SPSS - 22 (Statistical Package for the social sciences, 22.0 version) was used to interpret the data. Continuous variables are presented as mean $\pm \mathrm{SD}$ and categorical variables are expressed as frequencies and percentages. The pearson's chisquare test or the Fisher's exact test was used to determine if there is a relationship between two categorical variables $(p<0.05$ is considered to be significant).

\section{Results}

This was a prospective observational study. A total of 53 patients were included in this study, out of which $37(69.8 \%)$ were males and $16(30.2 \%)$ were females. Age of patients ranged from 14 years to 80 years with a mean age of $46.04 \pm 16.23$ years. Twenty eight patients $(52.8 \%)$ were agricultural workers and sustained injury while working on farms by vegetative matter. Forty nine $(92.45 \%)$ patients were from the rural areas with rural to urban ratio of $12: 1$. There were 22 patients $(41.5 \%)$ where primary treatment was started at our center, rest were already on treatment at the time of consultation.

Risk factors predisposing for fungal ulcer were present in half of the patients $(52.83 \%)$ (Fig. 1). History of trauma with some vegetative matter which included sugarcane leaf or rice barn or wood stick was present in $52.83 \%$ patients. Three patients $(5.66 \%)$ had underwent intraocular surgery after which they were on steroid treatment. Eight $(15.09 \%)$ patients had history of trauma due to other modes like, working in industry with machine or due to sand going into the eye. Fourteen patients $(26.41 \%)$ gave no history of trauma (Fig. 1).
Eighteen patients $(33.96 \%)$ had the onset of symptoms and signs in the months of July-September. Moderate grade of ulcer was seen in 39 patients $(73.58 \%)$. Nine $(16.98 \%)$ had mild and five $(9.44 \%)$ had severe grade of ulcer.

Signs and symptoms suggestive of typical fungal ulcer were noted in all patients. Fifty $(94.34 \%)$ patients in our study had serrated margins with raised slough. Ten patients $(18.87 \%)$ presented with satellite lesions. Forty five $(84.91 \%)$ patients had dry looking ulcer with cheesy infiltrates. Only four $(7.55 \%)$ patients had endothelial plaque. Nineteen patients (35.8\%) had presence of hypopyon where 15 had an immobile hypopyon and 4 had mobile hypopyon.

Out of 53 patients, $\mathrm{KOH}$ and culture was positive in $50.9 \%$ patients, while $18.9 \%$ were mixed type having both $\mathrm{KOH}$ and Gram stain positive. Total 27 patients were culture positive $(50.94 \%)$. The most common organism was Fusarium in 10 patients (18.9\%), followed by Curvularia in 8 patients (15.1\%), Bipolaris in 4 patients $(7.5 \%)$, Aspergillus in 3 patients $(5.7 \%)$, aureobasidium in 1 patient $(1.9 \%)$ and Yeast in 1 patient $(1.9 \%)$

There was a significant association between number of clinical features and $\mathrm{KOH}$ positivity. Similarly there was a significant association between no. of clinical features and culture positivity $(\mathrm{p}<0.001)$ (Table 1 and Table 2).

Out of fifty three patients, three were lost to follow up $(5.6 \%)$. Seventeen healed completely $(32.1 \%)$, two patient got perforated and BCL with glue was applied $(3.8 \%)$, twenty two are still on treatment $(41.5 \%)$ and nine patients had non-healing ulcer and had to undergo therapeutic keratoplasty $(17 \%)$.

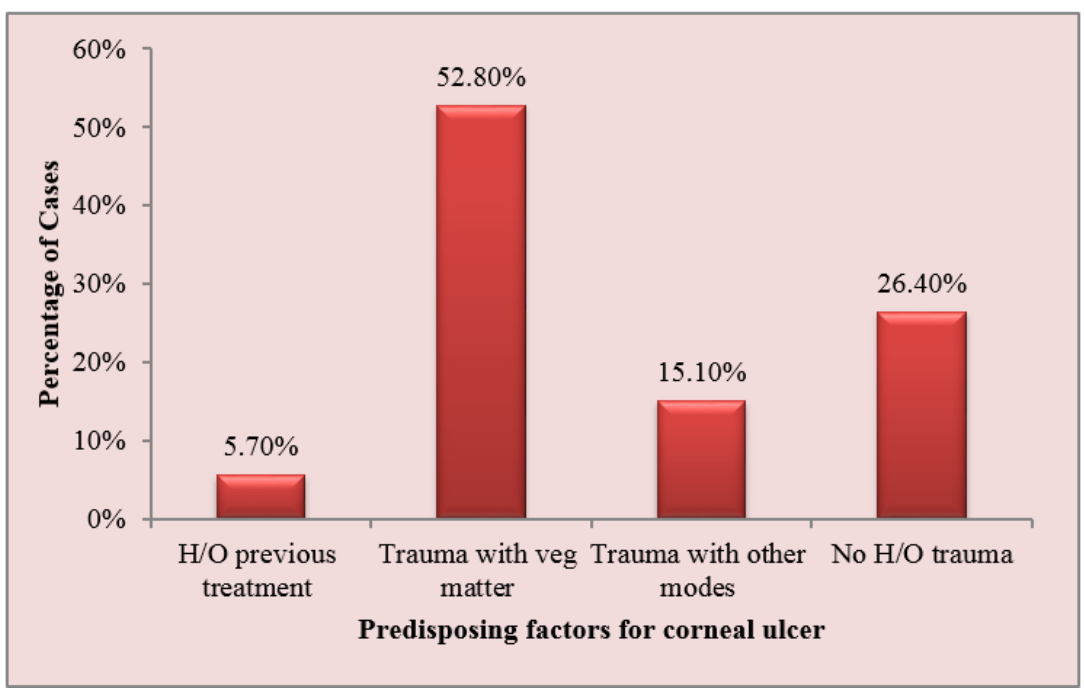

Fig. 1: Predisposing factors for corneal ulcer in the study group $(n=53)$ 
Table 1: Association between clinical features and $\mathrm{KOH}$ mount positivity in the study group (n=53)

\begin{tabular}{|l|c|c|c|c|c|}
\hline \multirow{2}{*}{$\begin{array}{l}\text { Number Clinical } \\
\text { Features Present }\end{array}$} & \multicolumn{2}{|c|}{ KOH Negative } & \multicolumn{2}{c|}{ KOH Positive } & \multirow{2}{*}{ p value } \\
\cline { 2 - 5 } & $\mathrm{n}$ & $\%$ & $\mathrm{n}$ & $\%$ & \\
\cline { 1 - 5 } & 23 & $88.5 \%$ & 0 & $0.0 \%$ & \\
\cline { 1 - 5 } & 0 & $0.0 \%$ & 4 & $14.8 \%$ & \multirow{2}{*}{$<0.001$} \\
\cline { 1 - 5 } & 0 & $0.0 \%$ & 17 & $63.0 \%$ & \\
\cline { 1 - 5 } & 0 & $0.0 \%$ & 4 & $14.8 \%$ & \\
\hline Total & 3 & $11.5 \%$ & 2 & $100 \%$ & \\
\hline
\end{tabular}

Fisher exact test

Table 2: Association between clinical features and culture positivity in the study group $(n=53)$

\begin{tabular}{|c|c|c|c|c|c|}
\hline \multirow{2}{*}{$\begin{array}{c}\text { Number of Clinical } \\
\text { Features Present }\end{array}$} & \multicolumn{2}{|c|}{ Culture Negative } & \multicolumn{2}{|c|}{ Culture Positive } & \multirow{2}{*}{ p value } \\
\hline & $\mathrm{n}$ & $\%$ & $\mathrm{n}$ & $\%$ & \\
\hline 1 & 23 & $88.5 \%$ & 0 & $0.0 \%$ & \multirow{6}{*}{$<0.001$} \\
\hline 2 & 0 & $0.0 \%$ & 4 & $14.8 \%$ & \\
\hline 3 & 0 & $0.0 \%$ & 17 & $63.0 \%$ & \\
\hline 4 & 0 & $0.0 \%$ & 4 & $14.8 \%$ & \\
\hline 5 & 3 & $11.5 \%$ & 2 & $7.4 \%$ & \\
\hline Total & 26 & $100 \%$ & 27 & $100 \%$ & \\
\hline
\end{tabular}

Fisher exact test

\section{Discussion}

Corneal infection is a leading cause of ocular morbidity and blindness worldwide. ${ }^{7}$ Mycotic keratitis is an infection caused by fungus that leads to inflammation and ulceration, usually following trauma or treatment for a bacterial infection with steroids or antibiotics. ${ }^{8}$ Inspite of the fast developing medical field it seems to be one of the greatest challenges to the ophthalmologists.

In our study, males were more commonly affected and both the sexes developed corneal ulcers in middle decades of their life. Significant male preponderance has been published in most of the previous studies available in the literature. Considering the predominant predisposing factor of trauma while outdoors, the probable reason for male majority is obvious. Similar results were seen in a study by Saha et al, where out of 65 patients, males were more affected $(76.1 \%)$. Most of them were in the age group of 41-60 years (71.4\%). Trauma was the most common predisposing factor $(80.9 \%)$ especially in agricultural workers and farm laborers $(76.1 \%){ }^{9}$

Ocular trauma was significantly higher and more associated with outdoor activities in the present study. Most of the patients were agricultural workers (52.8\%) from rural areas $(92.45 \%)$ and sustained injury with vegetative matter $(52.83 \%)$, predominantly with sugarcane leaf as sugarcane farming is common in this region. Thirty one patients $(58.5 \%)$ had taken prior treatment and had longer duration of symptoms. All these patients had taken mixed treatment from elsewhere and were not responding. Similar results were found in a study of Hyderabad by Gopinathan et al, where Odds ratio (OR) revealed that patients involved in agriculture-based activities were 1.33 times at a greater risk of developing microbial keratitis. ${ }^{10}$

In the present study, maximum patients $(33.96 \%)$ had onset of symptoms in July-September. A high prevalence of fungal keratitis in hot and humid climates has been widely reported in various studies. The incidence of fungal keratitis was higher during paddy harvesting and also during the time of year when agriculture activity was greater. A hot, humid, windy climate and an agriculture-based occupation of a large population make fungal keratitis more frequent in tropical zones. This was similar to a study conducted by Bharati et al, in South India, where fungal keratitis was seen more often between June - September. ${ }^{11}$

The typical features of fungal keratitis like serrated margins, raised slough, dry texture, cheesy infiltrates, satellite lesions, fixed hypopyon and pigmentation were the most common clinical features in our study. Hypopyon was present in 19 patients (35.8\%). Fixed hypopyon was seen in 15 out of 19 patients. Vascularisation of cornea was seen in six (11.3\%) patients. We found that out of 27 culture positive patients, 23 had presence of 3 or more typical clinical features of fungal keratits. There was significant association between number of clinical features and culture positivity $(\mathrm{p}<0.001)$. There has been a study by Thomas et al, where they found that serrated margins, raised slough and color were independently associated with fungal keratitis, when incorporated into a backwards stepwise logisitic regression model. The probability of fungal infection if one clinical feature was present was $63 \%$, increasing to $83 \%$ if all three features were present in their study. ${ }^{12}$

In our study, positive $\mathrm{KOH}$ were yielded in $50.9 \%$ patients and all of them were culture positive also. Our study had maximum of Fusarium (18.9\%) isolates 
followed by Curvularia (15.1\%), Bipolaris (7.5\%) and Aspergillus $(5.7 \%)$. In a study by Reema et al, in Assam, fungal element could be demonstrated in $65.2 \%$ cases in direct potassium hydroxide $(\mathrm{KOH})$ mount. Fusarium solani (25\%) was the commonest isolate followed by Aspergillus species (19\%), Curvularia species (18.5\%) and Penicillium species (15.2\%). Yeasts were isolated in $2.7 \%(n=5)$ cases. $^{13}$

There was no significant association between prior treatment taken with the $\mathrm{KOH}$ and culture positivity in our study. Therefore, we believe that despite the patient being on prior therapy, microbiological investigations may succeed in establishing etiological diagnosis in majority cases.

\section{Conclusion}

Corneal ulcer remains a cause for concern among the ophthalmologists in Garhwal region and predominance of agricultural activity in this region is the major risk factor. This geographic profile is vital for empirical management and diagnosis of keratitis where microbiological lab facilities are not available. It also helps us in formation of protocol and establishing guidelines for the same in population at risk.

\section{References}

1. Garg P, Krishna PV, Stratis AK, Gopinathan U. The value of corneal transplantation in reducing blindness. Eye 2005;19(10):1106-14.

2. Whitcher JP, Srinivasan M, Upadhyay MP. Corneal blindness: a global perspective. Bull World Health Organ. 2001;79(3):214-21.

3. Dandona R, Dandona L. Corneal blindness in a southern Indian population: Need for health promotion strategies. Br J Ophthalmol 2003;87(2):133-41.
4. Gopinathan U, Garg P, Fernandes M, Sharma S, Athmanathan S, Rao GN. The epidemiological features and laboratory results of fungal keratitis: a 10 -year review at a referral eye care center in South India. Cornea 2002;21(6):555-9.

5. Leck AK, Thomas PA, Hagan M, Kaliamurthy J, Ackuaku E, John M, et al. Aetiology of suppurative corneal ulcers in Ghana and south India, and epidemiology of fungal keratitis. Br J Ophthalmol 2002;86(11):1211-5.

6. Jones DB. Decision making in management of Microbial keratitis, Ophthalmology 1981;88(8):814-20.

7. Vemuganti GK, Garg P, Gopinathan U, Naduvilath TJ, John RK, Buddi R et al. Evaluation of agent and host factors in progression of mycotic keratitis: A histologic and microbiologic study of 167 corneal buttons. Ophthalmology 2002;109(8):1538-46.

8. Reddy PS, Satyendran OM, Satapathy M, Kumar HV, Reddy PR. Mycotic keratitis. Invest Ophthalmol 1972; 20(3):101-8

9. Saha R, Das S. Mycological profile of infectious Keratitis from Delhi. Indian J Med Res 2006;123(2):159-64.

10. Gopinathan U, Sharma S, Garg P, Rao GN. Review of epidemiological features, microbiological diagnosis and treatment outcome of microbial keratitis. Experience of over a decade. Indian J Ophthalmol 2009;57(4):273- 9.

11. Bharathi MJ, Ramakrishnan R, Meenakshi R, Palaniappan R. Epidemiological characteristics and laboratory diagnosis of fungal keratitis. A three-year study. Indian J Ophthalmol, 2003;51(4):315-21.

12. Thomas PA, Leck AK, Myatt M. Characteristic clinical features as an aid to diagnosis of suppurative keratitis caused by filamentous fungi. $\mathrm{Br}$ J Ophthalmol. 2005;89(12):1554- 8 .

13. Nath RMycotic corneal ulcers in upper Assam. Indian J Ophthalmol. 2011;59(5):367-371. 